\title{
La valoración de documentos de archivo y los responsables de la construcción de la memoria en la Administración Pública Federal brasileña
}

\author{
Maria Ivonete GOMES Do NASCIMENTO \\ ivonetenascimento40@gmail.com \\ Eliane BRAGA DE OLIVEIRA \\ elianebo@unb.br \\ Universidad de Brasilia (Brasil). \\ Facultad de Ciencias de la Información
}

Recibido: Octubre 2014

Aceptado: Noviembre 2014

\begin{abstract}
Resumen: Este trabajo presenta un resultado parcial de investigación sobre la valoración de documentos de archivo llevada a cabo en la Administración Pública Federal brasileña (APF), más concretamente en los ministerios del Poder Ejecutivo federal, con sede en Brasilia. Su objetivo es registrar las vertientes teóricas de la valoración y su relación con la memoria, así como identificar en la legislación brasileña y en las normativas del Archivo Nacional a los responsables de la evaluación de documentos de archivo en la APF brasileña. Se analizar las referencias bibliográficas y la legislación en la materia, y se realizó una recopilación de datos para examinar el cumplimiento de los preceptos establecidos en la legislación. identificaron distintas propuestas de evaluación documental. Se observó que existe un debate acerca del papel del archivista como constructor de memoria en la práctica de la valoración. Se verificó también que la legislación y el Archivo Nacional atribuyen la responsabilidad de esa tarea a las Comisiones Permanentes de Valoración de Documentos (CPAD) de los órganos públicos.
\end{abstract}

Palabras clave: Administración Pública Federal; Archivología; Archivos; Memoria; Valoración de documentos

The archival appraisal and those responsibles the construction of memory in the Brazilian Federal Public Administration

\begin{abstract}
This paper presents partial results of research on archival appraisal held in the Brazilian Federal Public Administration (APF), more specifically in the ministries of the Federal Executive Branch based in Brasilia. Its purpose is to record the theoretical aspects of the archival appraisal and its relation to memory, and to identify the Brazilian legislation and regulations of the National Archives those responsible for the archival appraisal in Brazilian APF. Bibliographical references and legislation relating to the matter were analyzed. A survey data was conducted to verify the compliance of the rules established in the legislation. Different proposals of archival appraisal were identified. It was observed that there is a debate about the role of the archivist as a builder of memory in the practice of archival appraisal. It was also
\end{abstract}


observed that the legislation and the National Archives attach to the Permanent Committees of Documents Appraisal of the public agencies, the responsibility of doing this.

Keywords: Archival appraisal; Archival studies; Archives; Brazilian Federal Public Administration; Memory

\section{INTRODUCCIÓN}

Tal como muestra la literatura especializada de la archivología ${ }^{1}$, los archivos son tan antiguos como la escritura, tanto como las personas que cuidaban de los documentos. En opinión de Cook (1998: p. 140), los «archivos reales de Mesopotamia, de Egipto, de China y de la América precolombina» nacieron para preservar las evidencias de transacciones legales, comerciales, de los privilegios constituidos y de los recuerdos del pasado. Por cada acción de un productor documental nacen uno o más documentos como resultado de las actividades establecidas para el logro de los objetivos definidos.

La producción documental creció de forma continua, a la vez que el crecimiento de la población, la ampliación del papel del Estado y de la administración pública, así como los cambios económicos, políticos y sociales. Como consecuencia, surgió una gran preocupación en este ámbito sobre qué hacer con esa producción documental creciente. De esa forma, a mediados del siglo XX, se propuso la valoración de documentos de archivo como intervención necesaria para controlar el crecimiento exponencial de los archivos de gobierno.

Según Duchein (1993: p. 12) la proliferación de la producción de documentos alteró la responsabilidad de los archivistas, que empezaron a preocuparse también de las condiciones de creación de los documentos, su acumulación, transferencia a los archivos y valoración. Para Llansó I Sanjuan (1993: p. 16) la administración moderna requiere de los archivistas una respuesta de mayor calado respecto a los documentos contemporáneos. Con eso, obliga a esos profesionales a una mayor intervención en la gestión y el tratamiento de los documentos antes de que adquieran valor histórico.

La valoración de archivo se ocupa de atribuir valor a los documentos y de definir los plazos de retención y destino, con el fin de propiciar una reducción del volumen de documentos, con la preservación de los que se consideren relevantes. En la archivología existe un debate acerca de esa función archivística, con lo que pueden identificarse diferentes proposiciones teóricas de diversos autores de diversos países. Fenoglio (2012) destaca que la importancia de la valoración reside

\footnotetext{
${ }^{1}$ La archivología, también llamada archivística, es la disciplina que estudia las funciones del archivo, los principios y técnicas relacionadas con la producción, organización, almacenamiento, preservación y utilización de los archivos(Archivo Nacional de Brasil, 2005: p. 37)
} 
en la irreversibilidad de la decisión tomada y en que eso puede comprometer el futuro de las investigaciones históricas venideras.

Este trabajo presenta un resultado parcial de investigación sobre la valoración de documentos de archivo llevada a cabo en los ministerios del Poder Ejecutivo, con sede en Brasilia, que forman parte de la Administración Pública Federal brasileña (APF).La investigación se lleva a cabo en el curso de máster del Programa de Posgrado en Ciencias de la Información de la Facultad de Ciencias de la Información de la Universidad de Brasilia. Su objetivo es registrar las vertientes teóricas de la valoración, así como identificar en la legislación brasileña y en las normativas del Archivo Nacional la indicación de los responsables de la valoración de documentos de archivo en la Administración Pública Federal brasileña. También se analizó literatura especializada con el objetivo de identificar posiciones diferentes en cuanto al papel del archivista en la práctica de la valoración y su interferencia en la construcción de la memoria.Para la recopilación de datos se utilizó un cuestionario, que fue respondido por los responsables de las unidades de archivo de los ministerios que formaron parte del campo empírico de la investigación.

\section{VALORACIÓN DE DOCUMENTOS DE ARCHIVO}

Los registros documentales suelen ser preservados por razones administrativas y legales, para comprobar derechos, deberes, contratos, testamentos, tratados, testimonios, etc. Según Duranti (1994), «a través de los milenios, los archivos han representado, de manera alterna y acumulativa, los arsenales de la administración, del derecho, de la historia, de la cultura y de la información» (Duranti, 1994: p. 6).

En opinión de Delmas (2010), «la necesidad de prueba frente a la justicia fue, en la sociedad occidental, la primera razón de la conservación de larga duración de determinados documentos escritos: diplomas merovingios y carolingios, actos, títulos, etc.»(DELMAS, 2010: p. 21). Los documentos también se preservan con fines de rendición de cuentas, de garantías de transparencia respecto a las actividades de organizaciones o gobiernos. Duranti (1994) afirma que el deber de prestar cuentas es un viejo concepto derivado de las directrices del emperador Carlomagno, que, en el 806 d. C., escribía a los condes: «Leed esta carta muchas veces, y guardadla bien, para que vosotros y nosotros podamos usarla como prueba para ver si actuasteis o no en consonancia con lo que en ella está escrito» (Heywood apud Duranti, 1994: p. 6). Según esa autora, los registros sirven para rendir cuentas respecto al aspecto administrativo e histórico, además de poder servir de prueba.

De la misma manera, los documentos considerados sin utilidad para esos usos son seleccionados y eliminados. Antes de la segunda mitad del siglo XX, la administración y los responsables de los archivos eliminaban los documentos sin 
criterios claros. Un ejemplo de ello se observó en Inglaterra, donde hubo una selección de emergencia de documentos debida a la demanda de papel viejo, para la fabricación de munición durante la Segunda Guerra Mundial, una situación en que la British Records Association promovió un amplio llamamiento para que se donaran exclusivamente los registros menos valiosos(Brooks, 1940). Duchein (1993: p. 12) afirma que el gobierno francés publicaba listas de eliminaciones de documentos administrativos, ya desde la primera mitad del siglo XIX, aunque no estaba regulada la elección sobre qué documentos se conservarían.

A mediados del siglo XX, el debate en torno a los registros que debían ser preservados o eliminados cobró mayor importancia, también estimulado por la falta de espacio para almacenar los fondos documentales que no cesaban de crecer. Surgen diversas opiniones acerca de cómo tratar esa cuestión. En ese contexto se determina la evaluación de documentos de archivo.

La valoración archivística consiste en la acción de analizar el valor de los documentos, primarios y secundarios, y de decidir sus respectivos plazos de retención y su destino final, con base en los valores atribuidos, considerando el contexto de relación entre el organismo y los documentos (Couture, 2003). En el Dicionário de Terminologia Arquivística, publicado por la Asociación de Archivistas de São Paulo, el término evaluación se define como "proceso de análisis de archivos, que busca establecer su destino en consonancia con los valores que les sean atribuidos»(Camargo; Belloto, 1996: p. 11). El Consejo Internacional de Archivos (CIA), en su base de datos Multilingual Archival Terminology, define la valoración como un «proceso de análisis de documentos de archivo, que establece los plazos de retención y destino, en consonancia con los valores que les son atribuidos» (Consejo Internacional de Archivos, 2014), según el Dicionário Brasileiro de Terminologia Arquivística del Archivo Nacional de Brasil.

El término inglés appraisal significa:«The process of identifying materials offered to an archive that have sufficient value to be accessioned». En francés, évaluation se define como:

Processus de sélection des activités métier pour déterminer quels documents doivent être capturés et combien de temps il faut les conserver pour répondre aux besoins métier, aux exigences de responsabilité et aux attentes de la communauté (Consejo Internacional de Archivos, 2014).

El ICA registra dos significados para la valoración en español. En primer lugar evaluación, usado en Argentina y Uruguay, que consiste en «el proceso de asignar el valor de los documentos de archivo con el propósito de determinar el periodo y las condiciones de su conservación»(Consejo Internacional de Archivos, 2014), y en segundo lugar valoración, de uso en España, México, Centroamérica y Colombia, definido como: 
El proceso de determinar el valor de los documentos de archivo con el propósito de establecer el período de retención y disposición (destrucción o transferencia) así como los términos y condiciones de transferencia de los mismos del productor hacia el preservador (Consejo Internacional de Archivos, 2014).

En el glosario del Proyecto InterPARES (International Research on Permanent Authentic Record in Eletronic Systems) ${ }^{2}$, valoración significa «The process of assessing the value of records for the purpose of determining the length and conditions of their preservation». Según Lacombe Rocha, ese significado afecta a todas las actividades del proyecto, así como a los documentos publicados por él (Lacombe, 2011). En el diccionario del mismo Proyecto, además de la definición registrada en el glosario, el término aparece con otras acepciones, dentro de asuntos relacionados con archivología, biblioteconomía, museos, ciencias de la información, informática, artes y otros usos generales:

n., The process of assessing the value of records for the purpose of determining the length and conditions of their preservation.

n., Refers to the process of evaluating records to determine whether they are to be archived indefinitely, retained for a shorter period, or disposed of in some other way (sold, donated, destroyed, etc.).

n., The process of identifying materials to be preserved because of their enduring value, especially those materials to be physically transferred to an archives. [Archives]

n., A basic archival function of determining the eventual disposal of records based upon their archival value. Also referred to as evaluation, review,selection or selective retention. [Archives]

n., The process of determining the value and thus the disposition of records based upon their current administrative, legal, and fiscal use; their evidential and information value; their arrangement and condition; their intrinsic value; and their relationship to other records.[Archives]

[...] (Consejo Internacional de Archivos, 2014)

Cabe destacar que los términos valoración y selección son equivalentes en varios países. En Brasil, aunque en la década de 1980 esos términos tuvieran el mismo significado, se considera que la selección consiste en separar los documentos de valor permanente de los que serán eliminados, con base en criterios

2 Proyecto coordinado por la Universidad de la Columbia Británica (Canadá), con la participación de varios países, con el objetivo de desarrollar un conocimiento teórico y metodológico para la preservación a largo plazo de los documentos archivísticos digitales, y que en su segunda fase presentó como productos el glosario, el diccionario y las ontologías, entre otros. 
definidos en la evaluación y orientada por la tabla de retención (Archivo Nacional de Brasil, 2005).

\section{LAS VERTIENTES TEÓRICAS DE LA VALORACIÓN DE DOCUMENTOS}

En este tema no se presenta una revisión exhaustiva de la literatura sobre evaluación, pero sólo señalar los autores más presentes en la literatura archivística difundida en Brasil.

La literatura internacional sobre archivos registra diversas propuestas para la valoración de documentos de archivo. Terry Cook (1999) y Terry Eastwood (2003) identifican tres corrientes de pensamiento en la materia. Los autores en que se fijan son Jenkinson, Schellenberg y el propio Cook. En este estudio se identifican igualmente otras propuestas para la valoración, lo que pone de manifiesto que el debate sigue siendo tratado por los archivistas.

Algunos de los autores estudiados (Armando M. da Silva; Fernanda Ribeiro; Júlio Ramos; Manuel L. Real, 1999) entienden que en Estados Unidos la valoración de documentos de archivo tenía un carácter más administrativo y económico que archivístico, mientras que en Europa ese quehacer estaba más orientado hacia el interés de la investigación histórica. Según Duchein (1993: p. 13), los modelos nacionales de valoración están relacionados con las condiciones culturales, económicas, administrativas y psicológicas de cada país o grupo de países. Sin embargo, en su opinión, ningún país puede escapar de la definición de su propia doctrina acerca del asunto.

Hilary Jenkinson (1922: p. 137), autor del respetado A Manual of Archive Administration, defendía que no se debía permitir ninguna interferencia en los documentos tras su producción, pues los documentos eran subproductos espontáneos de la administración, testimonio puro de actos y actuaciones, siendo una prueba testimonial, imparcial y auténtica que sirve de fuente para la investigación histórica. Para él, toda valoración por parte del archivista es impropia y pone en peligro la inocencia de los documentos, además de que el archivista no debe participar en la destrucción de los registros. Si fuera necesaria, la eliminación de documentos debería estar a cargo del organismo productor.

Philip Brooks (1940), archivista estadounidense, propone que se promuevan métodos que tengan en cuenta los valores en relación con la institución productora de los documentos, las políticas y las normativas administrativas de ese productor, la historia administrativa, así como los que abarcan derechos. Los documentos que no contienen valor legal, administrativo o que no presenten interés histórico para la investigación deben ser eliminados.

En 1956, Teodore Schellenberg propone que la valoración de los documentos públicos modernos se base en valores primarios y secundarios. El valor primario es el que se les atribuye a los documentos en función del interés que presentan para el 
órgano productor, teniendo en cuenta su utilidad para fines administrativos, legales y fiscales. El valor secundario se atribuye a los documentos en función del interés que puedan tener para la entidad productora y para otros usuarios, con miras a su utilidad para fines distintos de los que motivaron originalmente su producción. El análisis de esa distinción se da en relación con dos aspectos: la prueba que ofrece sobre la organización y de su funcionamiento, y la información que contienen sobre personas, cosas y fenómenos con que la entidad haya trabado contacto.

En la década de 1990, Terry Cook creó la denominada macroappraisal (macroevaluación) junto con otros archivistas, en el Archivo Nacional de Canadá. Esa propuesta se basa en el valor social de las funciones desarrolladas por el organismo productor del fondo que va a ser evaluado. Analiza el contexto de creación de los registros documentales y cómo son usados por sus productores en la interrelación con los ciudadanos, con los grupos y con las organizaciones en la contemporaneidad (COOK, 2006). La macroevaluación propone que los archivos reflejen la sociedad que los generó de forma holística, inclusiva, pasando de un discurso centrado solamente en el Estado hacia un discurso centrado igualmente en la sociedad, para reflejar su funcionamiento.

Luciana Duranti (1994), investigadora y autora de varios estudios archivísticos, profesora de la Universidad de la Columbia Británica (Canadá), destaca que en el debate del cambio de paradigma sobre la evaluación, con la excepción de Norteamérica, Alemania e Inglaterra, los demás países occidentales no se han manifestado sobre ese asunto.La autora sostiene que el desarrollo de la propuesta de atribuir valor a los documentos no vino precedida de la exploración del concepto de valoración, sino solo a partir de una reiterada repetición de la necesaria centralidad del trabajo archivístico en los tiempos modernos, y con eso busca su legitimación (DURANTI, 1994, p. 329).

Terry Eastwood (2003), asimismo profesor de la Universidad de la Columbia Británica, defiende la tesis de que el objetivo de la valoración depende del modelo de sociedad en la que se da la evaluación. En su opinión, en sociedades plurales debe darse también una pluralidad en los sistemas de archivos. El desafío es la elección de unos métodos que permitan a los miembros de una sociedad democrática preservar las fuentes para comprender el pasado y los caminos recorridos.

Jean-Yves Rousseau (1998), director del Servicio de Archivo de la Universidad de Montreal, y Carol Couture (1998), profesora de esa misma universidad, proponen la archivística integrada como la única capaz de garantizar una política completa de organización de los archivos, permitiendo un rápido acceso a las informaciones mediante procedimientos integrados de clasificación, valoración y descripción. La valoración se propone como función en los archivos corrientes e intermediarios.

Los investigadores portugueses Armando Malheiros Silva y Fernanda Ribeiro (2000) proponen la evaluación como una parte del polo técnico, de un método denominado «modelo cuadripolar». Esa metodología está constituida por los polos epistemológico, teórico, técnico y morfológico. Silva y Ribeiro (2004: p. 31; 2009, 
p. 288) proponen criterios y parámetros para la práctica de la valoración. Profundizan esa propuesta entendiendo la valoración como una operación metodológica aplicable a la información en cualquier contexto de producción y de uso. Esa propuesta de valoración establece tres tipos de indicadores: pertinencia, densidad y frecuencia.

Manuel Vázquez Murillo (2006), archivista argentino, afirma que el documento de archivo nace vinculado a una tramitación y establece la vigencia como centro del ciclo vital de los documentos archivísticos. La vigencia del documento está vinculada a su fuerza de obligar, testificar o probar, por lo que nada vigente debe ser eliminado. Entre el fin de la vigencia y la eliminación o remisión de los documentos a los archivos permanentes, transcurre un período de tiempo llamado plazo de precaución, cuyo objetivo radica en:

1. Responder a posibles reclamaciones administrativas o jurídicas sobre aspectos referidos a lo que dice el texto, o a su tramitación y cumplimiento.

2. Servir de antecedente directo o soporte jurídico a un documento que lo requiere para estar vigente. (Vázquez Murillo, 2006: p. 35)

Así, estos estudiosos proponen diversas miradas en relación con la valoración de documentos de archivo. Como apunta Fenoglio (2013: p. 1), no existe, en el ámbito internacional, un acuerdo total acerca de los criterios que deban ser adoptados en esa función archivística.

\section{LA VALORACIÓN Y EI ARCHIVISTA COMO AGENTE EN LA CONSTRUCCIÓN DE LA MEMORIA}

Para la mayoría de los estudiosos de la archivística, la valoración de documentos tiene como finalidad la selección y la adecuación de las fuentes como vestigios del pasado (Eastwood, 2003). Tal como observa Eastwood, esos estudios están orientados a conservar los documentos y a fomentar la memoria, con miras a la comprensión de ese pasado.

En opinión de Le Goff (1986: p. 535), esos documentos forman parte, junto con los monumentos, de los materiales de la memoria colectiva. Con relación a la revolución documental ocasionada por la ampliación del término documento, Le Goff observa:

A revolução documental tende também a promover uma nova unidade de informação: em lugar do fato que conduz ao acontecimento e a uma história linear, a uma memória progressiva, ele privilegia o dado, que leva a série e a uma história descontínua. Tornaram-se necessários novos arquivos, onde o primeiro lugar é ocupado pelo corpus, a fita magnética. A memória coletiva valoriza-se, institui-se em patrimônio cultural. O novo documento é 
armazenado e manejado nos bancos de dados. Ele exige uma nova erudição que balbucia ainda e que deve responder simultaneamente às exigências do computador e à crítica da sua sempre crescente influência sobre a memória coletiva. (Le Goff, 1986: p. 542)

Sin embargo, Le Goff alerta sobre el hecho de que el documento no es cualquier cosa que se quede en el pasado, sino que es, sobre todo, el resultado de la producción de la sociedad y de las relaciones de poder que en ella se dan. Lo que queda no es el conjunto de lo que existió en el pasado, sino una selección de las fuerzas que actúan en el momento de la selección de lo que será preservado (Le Goff, 1986).

Colombo (1991) apunta la estrecha relación entre el archivo y el olvido, afirmando que el motivo de la organización y de la racionalización del archivo busca suplir el extravío de los documentos y la pérdida del recuerdo. Colombo afirma que el olvido presenta dos aspectos: uno relativo «a la elección del material a ser traducido en recuerdos», al decidir ante un conjunto de informaciones que deben ser recordadas u olvidadas; y otro asociado a la «permanencia del recuerdo en su "lugar" mental o físico» (Colombo, 1991: p. 89).

Para Pollak (1989: p. 9), la memoria se integra en intentos de definir y de reforzar sentimientos de pertenencia y fronteras sociales entre colectividades. La referencia al pasado contribuye a la cohesión de los grupos, así como de las instituciones en la sociedad. De la misma forma, la memoria proporciona sentido de identidad individual y grupal. En su opinión:

A memória é, em parte, herdada, não se refere apenas à vida física da pessoa. A memória também sofre flutuações em função do momento em que ela é articulada, em que ela está sendo expressa. As preocupações do momento constituem um elemento de estruturação da memória (Pollak, 1992: p. 4).

Para este autor la memoria es selectiva, pues no todo queda grabado o registrado, es decir, ella y el documento son socialmente construidos. Asimismo, la memoria y la identidad son disputadas por los conflictos sociales e intergrupales. Pollak (1992) dice también que «el trabajo de encuadramiento de la memoria se alimenta del material suministrado por la historia». Sin embargo, el encuadramiento de la memoria grupal tiene límite y no puede ser construida de forma arbitraria, razón por la cual se da el suministro de referencias y de puntos de referencia. Pues «toda organización política [...] transmite su propio pasado y la imagen que ella forjó para sí misma». No obstante, es necesario mantener coherencia respecto a las interpretaciones (Pollak, 1992: p. 9).

Oliveira y Rodrigues (2007) sugieren la posibilidad de que la valoración de documentos también representaría un encuadramiento de la memoria en una organización. Con el procedimiento de adopción de valores, a partir de diversos criterios definidos para la construcción de la memoria, el archivista selecciona lo que queda y lo que será olvidado. Quizá se derive de ello la concepción de que el archivista es un constructor de memorias. 
La participación del archivista en la valoración de documentos y, consecuentemente, su papel como creador de memoria ha provocado debates en esta área, con opiniones diversas al respeto.

En las lecturas realizadas se percibe que la función del archivista también ha cambiado con la dinámica de la sociedad. En su origen, el profesional de archivo era el responsable de los documentos y llegaba a ser confundido con el escriba del antiguo Egipto, persona de confianza del poder. Posteriormente, surge el archivista paleógrafo del siglo XIX, del École des Chartes, que almacenaba los documentos procedentes de las secciones en que se instruían los procesos, con vistas a que sirvieran para la investigación histórica. Del archivista erudito, organizador y animador (DELMAS, 2010), pasando por el profesional pasivo y sin ninguna interferencia en relación con la valoración de Jenkinson; del archivista que ejerce un papel decisivo en decisiones sobre la preservación y la eliminación de los documentos de Schellenberg, al archivista mediador activo del pasado de Cook.

Con la macroevaluación propuesta por Cook (1999: p. 2), el archivista sigue asumiendo un papel destacado en la preservación y eliminación documental. Para ese autor «appraisal imposes a heavy responsibility on archivists». Teniendo en cuenta su papel en ese proceso:

We are literally creating archives. We are deciding what is remembered and what is forgotten, who in society is visible and who remains invisible, who has a voice and who does not (Cook, 1999: p. 2).

Cook cuestiona el papel del archivista en la definición de la memoria, «¿por qué adoptamos una mitología profesional de almacenamiento pasivo y no de mediación activa con el pasado?»(Cook, 2012: p. 144), y teje las siguientes observaciones:

In this acto of creation, we must remain extraordinarily sensitive to he political, social, and philosophical nature of documents individually, of archives collectively, of archival functions, of archivists' personal bias, and most especially of archival appraisal, for that process defines the creators, functions, and activities to be reflected in archives, by defining, choosing, selectin which related documents are to be preserved permanently, and thus are to enjoy all subsequent archival processes (description, conservation, exhibition, reference, etc.), and, as starkly, and with finality, which are destroyed, excluded from archives, forgotten from memory.(Cook, 1999: p. 2)

Schwartz y Cook (2004: p. 15) también resaltan que los archivistas, en cuanto gestores de archivos, ostentan un poder en relación con los documentos que constituirán la memoria y la identidad: 
são vitais para o entendimento da natureza dos arquivos enquanto instituições e enquanto lugares de memória social. Tais contextos também influenciam os arquivos no nível individual da criação e da sobrevivência de $\mathrm{u}[. .$.$] os princípios e as estratégias que os arquivistas adotam ao passar do$ tempo, e as atividades que desenvolvem - especialmente selecionar e avaliar o que se tornará de guarda permanente e o que será descartado - influenciam a natureza e a ordenação dos conteúdos arquivísticos e, dessa forma, a memória da sociedade. Estes contextos culturais subjacentes $\mathrm{m}$ único documento: a carta, a fotografia, o diário, o vídeo caseiro. Como os arquivos coletivamente, um documento individual não é somente portador de conteúdo histórico, mas, também um reflexo das necessidades e desejos do seu produtor, dos propósitos de sua criação, do seu usuário, do alcance legal, técnico, organizacional, social, e cultural-intelectual com o qual o produtor e o usuário operam, e no qual o documento tem significado, e a intervenção inicial e a mediação contínua dos arquivistas. (Schwartz; Cook, 2004: p. 16)

Según Cox (2003), los archivistas deben considerar las implicaciones de la memoria colectiva, en la función de la valoración de archivos. Debido a esa acción, se forman los archivos y su utilidad para la sociedad. Para él, la memoria colectiva proporciona un contexto para la comprensión de la naturaleza de los archivos, como lugares de esas memorias.

Couture (2003) observa que para el trabajo de la determinación del valor documental, el archivista se debe asegurar al desarrollar la valoración y prestar atención a los cinco principios básicos aplicables a todos los contextos, aquí citados:

- De que los archivos den testimonio del conjunto de actividades de la sociedad;

- De la objetividad y la contemporaneidad del criterio que adopta;

- De respetar los nexos de unión entre la valoración y las demás intervenciones archivísticas;

- De que, en su intervención, exista equilibrio entre los fines administrativos y los fines patrimoniales;

- De que exista equilibrio entre las consideraciones relativas al contexto de creación de los documentos y las relativas a su uso(Couture, 2003: p. 31).

En opinión de Delmas (2010), las tareas del archivista deben quedar fuera de cualquier influencia de tendencias u otras preocupaciones que no sean su recogida en los archivos, la selección y la elaboración de los instrumentos de investigación. El archivista reconstituye el contexto de los documentos y los hace accesibles para las investigaciones, concibe y organiza el almacenamiento y promueve la valorización científica e histórica de la memoria social.Por eso, para el autor, ese profesional necesita ser neutro y objetivo en relación con la aplicación de las reglas. 
Se observa que no existe un consenso de los autores respecto al papel desempeñado por el archivista en la construcción de la memoria de la sociedad al realizar la valoración de documentos de archivo.Sin embargo, no es posible ignorar que la subjetividad presente en el proceso de elección interfiere en la construcción de un fondo que prioriza una determinada mirada.

\section{LOS RESPONSABLES DE LA VALORACIÓN DOCUMENTAL EN LA ADMINISTRACIÓN PÚBLICA FEDERAL BRASILEÑA}

La valoración de documentos de archivo está prevista en la legislación federal brasileña a partir de la ley n. ${ }^{\circ} 8.159$, conocida como «Ley de Archivos», de 8 de enero de 1991. En consonancia con la Constitución Federal de 1988, ella reconoce la gestión de documentos y la protección de los documentos de archivo como un deber de los poderes públicos. La valoración de documentos se entiende como parte de la gestión documental, según el art. 3.':

Considera-se gestão de documentos o conjunto de procedimentos e operações técnicas referentes à sua produção, tramitação, uso, avaliação e arquivamento em fase corrente e intermediária, visando a sua eliminação ou recolhimento para a guarda permanente(Brasil, 1991).

El Decreto n. ${ }^{\circ} 4.073 / 2002$, que regula la Ley n. ${ }^{\circ} 8.159 / 1991$, al tratar de la gestión de documentos de la Administración Pública Federal, crea la obligatoriedad para todos los órganos públicos de constituir una Comisión Permanente de Evaluación de Documentos (CPAD):

Art.18. Em cada órgão e entidade da Administração Pública Federal será constituída comissão permanente de avaliação de documentos, que terá a responsabilidade de orientar e realizar o processo de análise, avaliação e seleção da documentação produzida e acumulada no seu âmbito de atuação, tendo em vista a identificação dos documentos para guarda permanente e a eliminação dos destituídos de valor.

$\S 1^{\circ}$ Os documentos relativos às atividades-meio serão analisados, avaliados e selecionados pelas Comissões Permanentes de Avaliação de Documentos dos órgãos e das entidades geradores dos arquivos, obedecendo aos prazos estabelecidos em tabela de temporalidade e destinação expedida pelo CONARQ. $\S 2^{\circ}$ Os documentos relativos às atividades-meio não constantes da tabela referida no $\S 1^{\circ}$ serão submetidos às Comissões Permanentes de Avaliação de Documentos dos órgãos e das entidades geradores dos arquivos, que estabelecerão os prazos de guarda e destinação daí decorrentes, a serem aprovados pelo Arquivo Nacional.

$\S 3^{\circ}$ Os documentos relativos às atividades-fim serão avaliados e selecionados pelos órgãos ou entidades geradores dos arquivos, em 
conformidade com as tabelas de temporalidade e destinação, elaboradas pelas Comissões mencionadas no caput, aprovadas pelo Arquivo Nacional (Brasil, 2002).

Ese mismo decreto establece que los documentos de archivo, en el ámbito del gobierno federal, tendrán que ser evaluados, organizados, higienizados y acondicionados al ser transferidos o recogidos en el archivo nacional.

La Resolución $n^{\circ}$. 14, del Consejo Nacional de Archivos-CONARQ, de 24 de octubre de 2001, adopta las disposiciones relativas a los períodos de custodia y disposición de los documentos establece una tabla básica de temporalidad y eliminación de documentos de archivo a los órganos de la administración pública. Esta resolución reafirma que la eliminación de documentos producidos por las instituciones públicas se realizará después de autorización por los archivos nacionales.

La eliminación de documentos en agencias gubernamentales sólo puede ocurrir después de la terminación del proceso de evaluación, implementado por las comisiones permanentes de evaluación. La institución deberá cumplir con la publicación de eliminación, como se prevé en la Resolución n. ${ }^{\circ} 5$, de 30 de septiembre de 1996. Asimismo, la Resolución n. ${ }^{\circ}$ 7, de 20 de mayo de 1997, determina la lista de documentos para ser eliminados en su listado y el término de eliminación con respecto a la ley en sí.

Los documentos digitales están cubiertos por la resolución $\mathrm{n}^{\circ} 20$, de 16 de julio de 2004. La gestión de archivo de documentos digitales deberá adoptar los mismo procedimientos y criterios para la evaluación y la práctica de destino documental, citado en la anterior legislación. La eliminación de estos documentos debe preverse en la tabla de la temporalidad.

Lo Decreto $\mathrm{n}^{\circ}$ 4.915, de 12 de diciembre de 2003 crea lo Sistema de Gestión de Documentos de Archivo - SIGA. Entre otros fines, SIGA debe cuidar a los registros de racionalización de la producción en el sector público, que incluye los documentos de evaluación.

Según la resolución $\mathrm{N}^{\mathrm{o}} 24$, del 03 de agosto de 2006, los documentos de archivo digital y digital no forman parte del conjunto ser transferidos y recogidos por instituciones públicas, deben ser previamente identificados, clasificados, evaluados y asignado. Esta predicción se está determinada por la tabla de temporalidad y eliminación de documentos o de destino. Deben también ser aprobados por las instituciones archivísticas en su esfera de actividad.

E-ARQ Brasil adopta el modelo de requisitos para sistemas informatizados de gestión archivística de documentos-SIGAD, en conformidad con la resolución no. 25, de 27 de abril de 2007, a todos los órganos y entidades del sistema nacional de archivos. Establece varias condiciones que deben cumplir por estas organizaciones, sistemas y también por el documento, con el fin de garantizar la confiabilidad, autenticidad y acceso a los registros. Tiene como objetivo orientar e 
implementar la gestión documental a la provisión de las especificaciones técnicas y funcionales, además de los metadatos de sistemas informatizados de reserva. Para e-Arq Brasil evaluación se define como:

Um processo de análise dos documentos arquivísticos, visando os prazos de guarda e a destinação, de acordo com os valores primários e secundários que lhes são atribuídos. Os prazos de guarda e as ações de destinação deverão estar formalizados na tabela de temporalidade e destinação do órgão ou entidade. Os prazos de guarda referem-se ao tempo necessário para o arquivamento dos documentos nas fases corrente e intermediária, visando atender, exclusivamente, às necessidades da administração que os gerou, baseado em estimativas de uso (Conselho Nacional de Arquivos, 2011: p.30).

Retrocediendo en el tiempo, es posible observar que algunas iniciativas del Archivo Nacional de Brasil influyeron no solo en la promulgación de la Ley de Archivos, sino también en la práctica archivística llevada a cabo en los órganos de la Administración Pública Federal.

En 1985, el Archivo Nacional editó una publicación técnica, titulada Orientação para avaliação e arquivamento intermediário em arquivos públicos (Orientación para valoración y archivo intermediario en archivos públicos).Esa publicación supuso una primera orientación en materia de valoración de documentos para los archivos público, y estableció directrices generales para la elaboración de tablas de retención.En su texto, la evaluación se define como el acto de establecer principios orientadores para el análisis y la selección de documentos, con la finalidad de constituir plazos de retención y definir un destino final para los documentos.

De la misma manera, orienta la composición del equipo técnico encargado de hacer la valoración de documentos de archivo.

Ese grupo debe estar formado por: un archivista o un responsable del almacenamiento de documentos; una autoridad administrativa, con conocimiento de la estructura y el funcionamiento del órgano; un profesional del ámbito jurídico y profesionales del campo de conocimiento de que trata el fondo.

Para la identificación del valor de los documentos, sugiere que se respondan algunas indagaciones. En relación con el valor administrativo, debe verificarse la frecuencia de consulta del documento, ya sea el original o el que contenga información acerca de la historia del órgano. En la identificación del valor histórico, debe evaluarse la información referente a asuntos de interés para investigadores, tales como: información administrativa, política, científica, tecnológica y cultural, que pueda reconstituir la memoria nacional.

El Archivo Nacional sugiere como documentos de almacenamiento temporal los que contengan informaciones de interés meramente administrativo, como:

a. Cuyos textos estén reproducidos en otros; 
b. Cuyos textos hayan sido impresos en su totalidad;

c. Cuyos elementos esenciales se hallen recapitulados en otros;

d. Que presenten repetición de la información y escasa calidad técnica (en el caso de documentación especial);

e. Que sean copias y duplicados de originales destinados al almacenamiento permanente;

f. Que, aunque sean originales, tengan interés administrativo solo por un período determinado;

g. Sujetos a plazos de prescripción (Archivo Nacional de Brasil, 1985: p. 15).

Presenta también las características de los documentos de almacenamiento permanente:

a. [...] relativos al origen, a los derechos y a los objetivos del organismo y del órgano. [...]

b. [...] que reflejen la organización y el desarrollo del organismo y del órgano. $[\ldots]$

c. [...]visuales y/o sonoros referentes a hechos de la vida del organismo y del órgano. [...]

d. [...] que firman jurisprudencia. [...]

e. [...] relativos a la administración de personal. [...]

f. [...] que respondan a cuestiones técnico-científicas relacionadas con las actividades específicas del organismo y del órgano. [...]

g. [...] de divulgación o de promoción del organismo y del órgano, de los cuales debe ser almacenado por lo menos un ejemplar como muestra. [...]

h. [...] cuyas características extrínsecas sean de valor artístico y cultural (Archivo Nacional de Brasil, 1985: p. 17).

El documento indica que, para elaborar la tabla de retención, se debe observar la teoría de las tres edades y la identificación de los valores primarios y secundarios de los documentos, en función de su potencial de uso. La valoración también está relacionada con la depuración de la masa documental archivística, en que se eliminan los documentos que no contengan ningún valor y se conservan los que tengan valor para fines administrativos o de investigación histórico-científica.

Esas recomendaciones son de especial relevancia, puesto que guiaron las directrices establecidas para la APF, a partir de la promulgación de la Ley de Archivos y de toda la normativa derivada de su regulación. 
Tabla I. Resumen de la legislación sobre evaluación de documentos en la APF Fuente: Elaboración propia

\begin{tabular}{|c|c|c|}
\hline Norma & Asunto & Dispositivos \\
\hline $\begin{array}{l}\text { Ley } \mathrm{n}^{\circ} 8.159 \text {, de } 8 \\
\text { de enero de } 1991\end{array}$ & $\begin{array}{l}\text { Dispone acerca de la política nacional de archivos } \\
\text { públicos y privados y da otras providencias }\end{array}$ & Art. $3^{\circ}, 9^{\circ}$ \\
\hline $\begin{array}{l}\text { Decreto } n^{\circ} 4.073 \text {, de } \\
3 \text { de enero de } 2002\end{array}$ & Reglamenta la Ley no 8.159/1991 & $\begin{array}{l}\text { Art. } 18, \S 1^{\circ}, \\
\S 2^{\circ}, \S 3^{\circ}\end{array}$ \\
\hline $\begin{array}{l}\text { Decreto } n^{\circ} 4.915 \text {, de } \\
12 \text { de diciembre de } \\
2003\end{array}$ & $\begin{array}{l}\text { Dispone acerca del Sistema de Gestión de } \\
\text { Documentos de Archivos - SIGA, de la } \\
\text { Administración Pública Federal, y da otras } \\
\text { providencias }\end{array}$ & $\begin{array}{l}\text { Art. } 5^{\circ}, \mathrm{II}, \\
\text { IV,V }\end{array}$ \\
\hline $\begin{array}{l}\text { Resolución } n^{\circ} 5 \text {, de } \\
30 \text { de septiembre de } \\
1996\end{array}$ & $\begin{array}{l}\text { Prevé la publicación de avisos para la eliminación } \\
\text { de documentos en los boletines oficiales de la } \\
\text { Unión, Distrito Federal, los estados y municipios }\end{array}$ & Art. $1^{\circ}$ \\
\hline $\begin{array}{l}\text { Resolución } n^{\circ} 7 \text {, de } \\
20 \text { de mayo de } 1997\end{array}$ & $\begin{array}{l}\text { Dispone acerca de los procedimientos para la } \\
\text { eliminación de documentos en el ámbito de los } \\
\text { organismos y entidades partes del Poder Público }\end{array}$ & $\begin{array}{l}\text { Art. } 1^{\circ}, \quad 2^{\circ}, \\
3^{\circ}, 4^{\circ}, 5^{\circ}\end{array}$ \\
\hline $\begin{array}{l}\text { Resolución } \mathrm{n}^{\mathrm{o}} 14 \text {, de } \\
24 \text { de octubre de } \\
2001\end{array}$ & $\begin{array}{l}\text { Aprueba la versión revisada y ampliada de la } \\
\text { Resolución } \mathrm{n}^{\circ} 4 \text {, de } 28 \text { de marzo del 1996, que } \\
\text { dispone acerca del Código de Clasificación de } \\
\text { Documentos de Archivo para la Administración } \\
\text { Pública: Actividades-medio, a ser adoptado como } \\
\text { modelo para los archivos corrientes de los órganos } \\
\text { y entidades partes del Sistema Nacional de } \\
\text { Archivos - SINAR, y los plazos de guardia y la } \\
\text { destinación de los documentos establecidos en la } \\
\text { Tabla Básica de Temporalidad y Destinación de } \\
\text { Documentos de Archivo relativos a las } \\
\text { Actividades-medio de la Administración Pública }\end{array}$ & Art. $1^{\circ}, 2^{\circ}, 3^{\circ}$ \\
\hline $\begin{array}{l}\text { Resolución } n^{\circ} 20 \text {, de } \\
16 \text { de julio de } 2004\end{array}$ & $\begin{array}{l}\text { Dispone acerca de la inserción de los documentos } \\
\text { digitales en los programas de gestión de archivo } \\
\text { de documentos de los órganos y entidades parte } \\
\text { del Sistema Nacional de Archivos }\end{array}$ & $\begin{array}{l}\text { Art. } 1^{\circ}, \S 3^{\circ} ; \\
5^{\circ}\end{array}$ \\
\hline $\begin{array}{l}\text { Resolución } n^{0} 24, \text { de } \\
3 \text { de agosto de } 2006\end{array}$ & $\begin{array}{l}\text { Establece directrices para la transferencia y la } \\
\text { colección de documentos de archivo digitales a las } \\
\text { instituciones públicas de archivo }\end{array}$ & Art. $\left.1^{\circ}, \mathrm{a}\right)$ \\
\hline $\begin{array}{l}\text { Resolución } n^{\circ} 25, \text { de } \\
27 \text { de abril de } 2007\end{array}$ & $\begin{array}{l}\text { Dispone acerca de la adopción del Modelo de } \\
\text { Requisitos para Sistemas Informatizados de } \\
\text { Gestión Archivística de Documentos - e-ARQ } \\
\text { Brasil por los órganos y entidades partes del } \\
\text { Sistema Nacional de Archivos - SINAR }\end{array}$ & Art. $1^{\circ}, \S 1^{\circ}$ \\
\hline
\end{tabular}




\section{LAS COMISIONES PERMANENTES DE VALORACIÓN DE DOCUMENTOS EN LOS ÓRGANOS PÚBLICOS}

Teniendo en cuenta la obligatoriedad de constituir comisiones permanentes de valoración de documentos (CPAD) en los órganos públicos federales del Poder Ejecutivo, se realizó una recopilación de datos, mediante aplicación de cuestionarios, en los veinticuatro ministerios de la Administración Pública Federal, con sede en Brasilia. Son los siguientes:

Tabla II. Ministerios de la Administración Pública Federal brasileña Fuente: Elaboración propia

\begin{tabular}{l|l}
\hline \multicolumn{1}{c|}{ NOMBRE } & \multicolumn{1}{c}{ SIGLA } \\
\hline Ministerio de Relaciones Exteriores & Itamaraty \\
\hline Ministerio de Defensa & Defesa \\
\hline Ministerio de Cultura & Cultura \\
\hline Ministerio de Educación & MEC \\
\hline Ministerio de Integración Nacional & Integração \\
\hline Ministerio de Justicia & MJ \\
\hline Ministerio de Agricultura, Ganadería y Abastecimiento & MAPA \\
\hline Ministerio de Hacienda & Fazenda \\
\hline Ministerio de Comunicaciones & MC \\
\hline Ministerio de Ciencia y Tecnología e Innovación & MCTI \\
\hline Ministerio de Desarrollo, Industria y Comercio Exterior & MDIC \\
\hline Ministerio de Previsión Social & Previdência \\
\hline Ministerio de Turismo & Turismo \\
\hline Ministerio de Minas y Energía & MME \\
\hline Ministerio de Desarrollo Social y Combate al Hambre & MDS \\
\hline Ministerio de Planeamiento, Presupuesto y Gestión & MPOG \\
\hline Ministerio del Medioambiente & MMA \\
\hline Ministerio da Pesca y Acuicultura & MPA \\
\hline Ministerio de las Ciudades & Cidades \\
\hline Ministerio de Desarrollo Agrario & MDA \\
\hline Ministerio de Deportes & ME \\
\hline Ministerio de Trabajo y Empleo & TEM \\
\hline Ministerio de Transportes & Transportes \\
\hline Ministerio de Salud & Saúde \\
\hline
\end{tabular}

Realizada entre septiembre de 2013 y febrero de 2014, esta recopilación buscaba examinar el cumplimiento de esa directiva entre esos órganos del Poder Ejecutivo Federal. La encuesta ha sido aplicada en las visitas a los componentes del universo de búsqueda. Todos los veinticuatro ministerios han contestado. Los encuestados son los responsables de los sectores del archivo. Estos servidores, en su mayoría, son profesionales de nivel superior, siendo quince de ellos archivistas. 
Entre los otros son los bibliotecarios y administradores. Han sido identificados, también, tres servidores de nivel medio. Cabe destacar que los archivistas responsables de sectores de archivo son también miembros de los comités permanentes de evaluación de documentos impuestas en los ministerios.

A partir de esos datos, se observa que, pese a la determinación legal, algunos ministerios aún no han constituido sus Comisiones Permanentes de Evaluación de Documentos, aunque en su mayoría eso ya ocurra. Sin embargo, el Decreto 4.073/2002 está en vigor desde hace más de diez años, lo que hace difícil justificar el incumplimiento de ese precepto legal, según los siguientes datos.

\section{¿Se ha constituido formalmente la Comisión Permanente de Valoración de Documentos en el órgano?}

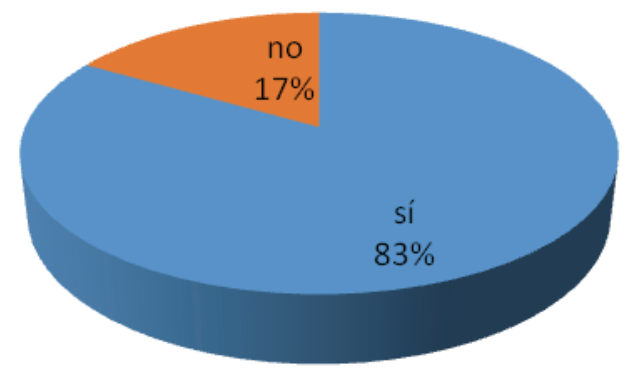

Fuente: Elaboración propia, con base en los datos obtenidos

Esas Comisiones Permanentes de Valoración de Documentos de Archivo tienen la responsabilidad de orientar y realizar la evaluación y selección de documentos de forma constante dentro del órgano. Para que eso ocurra es necesaria una CPAD con funcionamiento regular, con atribuciones claras y definidas. Sin embargo, en este estudio se ha observado que pocas comisiones han establecido un reglamento interno, lo que puede comprometer el funcionamiento regular de las comisiones. 


\section{¿Existe un reglamento interno de la Comisión \\ Permanente de Valoración Documental?}

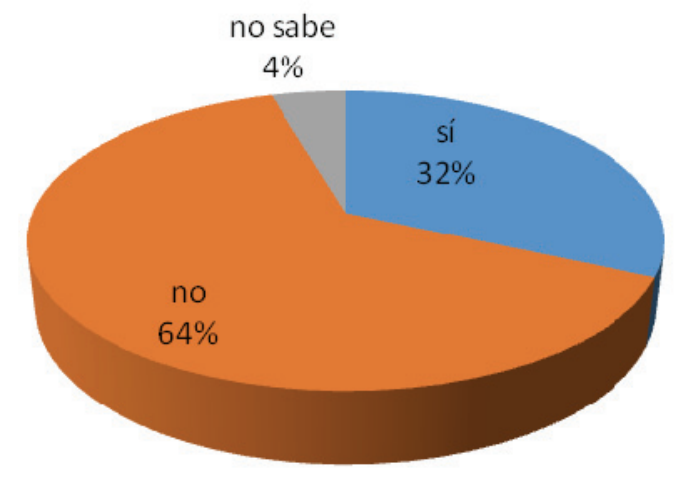

Fuente: Elaboración propia, con base en los datos obtenidos

\section{CONSIDERACIONES FINALES}

En la revisión bibliográfica sobre las vertientes teóricas de la valoración de documentos de archivo, se observa que los autores, en sus propuestas, parten de la constatación de la imposibilidad de almacenar toda la producción documental contemporánea. A partir de esa constatación, desarrollan criterios para definir qué consideran importante guardar como memoria de la sociedad y qué puede ser eliminado. Así, aparecen soluciones sobre cómo debe llevarse a cabo la evaluación, con la formulación de propuestas metodológicas de esa práctica archivística. Sin embargo, se observa que Schellenberg fue quien más influyó en la práctica archivística brasileña.

De la misma manera, se identifica un discurso en relación con la valoración documental, comprendiéndola como una tarea que interfiere en la construcción de la memoria. La preservación de los documentos se considera esencial para comprender mejor el pasado y servir de base para las elecciones del futuro. Sin embargo, no existe consenso entre los autores sobre el papel desempeñado por el archivista, en cuanto agente social que interfiere en la construcción de la memoria de la sociedad, al realizar la valoración de documentos. Algunos autores, en especial los vinculados al pensamiento llamado posmoderno, admiten la subjetividad inherente al proceso de elección en que está involucrado el archivista, que da lugar a la construcción de un fondo a partir de una determinada mirada, sobre una realidad dada. Con base en la representación resultante de esa construcción, se elaborarán nuevas memorias.

Se constató que la legislación brasileña y el Archivo Nacional atribuyen a las Comisiones Permanentes de Valoración de Documentos (CPAD) la 
responsabilidad de realizar la valoración, considerada indispensable para la preservación de la memoria archivística de las instituciones del poder público.

Las CPADs están formadas por funcionarios públicos. Son archivistas, bibliotecarios, administradores, abogados, servidores de los sectores de documentación e información y servidores de nivel medio. Esa comisión desempeña la función de realizar el proceso de valoración de los documentos producidos y acumulados por cada órgano. Sin embargo, no todos los ministerios han creado su CPAD. Entre las existentes, pocas cuentan con un reglamento interno, lo que sugiere una fragilidad en el desempeño de sus funciones, algo muy preocupante, puesto que desde el punto de vista legal las CPAD son las responsables de la valoración de documentos en los órganos del poder ejecutivo federal.

En Brasil, los archiveros participantes de las CPADs tienen su autonomía limitada al cumplimiento de lo que se espera en la legislación brasileña y en las directrices de lo Archivo Nacional. Incluso las tablas de documentos de actividades sustantivas, una de las tareas de CPADs, depende de la promulgación definitiva de lo Archivo Nacional. Sin duda, es evidente el papel destacado desempeñado por el Archivo Nacional de Brasil, con respecto a la disposición de los documentos públicos.

Se encontró que la legislación brasileña adopta los conceptos de valores primarios y secundarios para establecer el destino de los documentos. Algunas normas sugieren la integración de las fases corriente, intermedia y permanente, influencia de los autores Jean-Yves Rousseau y Carol Couture. El concepto de prazo precaucional de Manuel Vázquez se aplica en el Tabla Básica de Temporalidad y Documento de Archivos, de la Resolución n. ${ }^{\circ}$ 14/2001

Además de estas contribuciones, resulta evidente la influencia de la archivística americana en la legislación brasileña, en especial, la presencia determinante de las ideas de Brooks y Schellenberg, en lo tocante a la valoración de documentos, como evidencia en la tabla III.

Finalmente, conviene recordar que la valoración de documentos debe ser un procedimiento de carácter ordinario y sistemático para una gestión documental comprometida con la ciudadanía y la transparencia administrativa. 
Tabla III. Referencias conceptuales identificadas en la legislación brasileña

Fuente: Elaboración propia

\begin{tabular}{|c|c|c|}
\hline Norma & Dispositivos & $\begin{array}{l}\text { Autor da vertente da avaliação de } \\
\text { documento de arquivo identificada }\end{array}$ \\
\hline Lei $n^{\circ} 8.159 / 1991$ & Art. $3^{\circ}, 9^{\circ}$ & Teodore Schellenberg \\
\hline $\begin{array}{l}\text { Decreto } \mathrm{n}^{\circ} \\
4.073 / 2002\end{array}$ & $\begin{array}{l}\text { Art. } 18, \quad \S 1^{\circ}, \\
\S 2^{\circ}, \S 3^{\circ}\end{array}$ & Teodore Schellenberg \\
\hline $\begin{array}{l}\text { Decreto } \mathrm{n}^{\circ} \\
4.915 / 2003\end{array}$ & Art. $5^{\circ}, \mathrm{II}, \mathrm{IV}, \mathrm{V}$ & $\begin{array}{l}\text { Teodore Schellenberg } \\
\text { Jean-Yves Rousseau } \\
\text { Carol Couture }\end{array}$ \\
\hline Resolução no 5/1996 & Art. $1^{\circ}$ & Teodore Schellenberg \\
\hline Resolução no $7 / 1997$ & Art. $1^{\circ}, 2^{\circ}, 3^{\circ}, 4^{o}, 5^{\circ}$ & Teodore Schellenberg \\
\hline $\begin{array}{l}\text { Resolução n } \\
\text { 14/2001 }\end{array}$ & Art $.2^{o}, \S 1^{\circ}, 2^{o} ; 3^{o}$ & $\begin{array}{l}\text { Jean-Yves Rousseau } \\
\text { Carol Couture } \\
\text { Teodore Schellenberg } \\
\text { Manuel Vazquez }\end{array}$ \\
\hline $\begin{array}{l}\text { Resolução n } \\
\text { 20/2004 }\end{array}$ & $\begin{array}{l}\text { Art. } 1^{\mathrm{o}}, \S 3^{\mathrm{o}} ; 3^{\mathrm{o}}, \S 1^{\mathrm{o}} ; \\
5^{\mathrm{o}}\end{array}$ & $\begin{array}{l}\text { Teodore Schellenberg } \\
\text { Jean-Yves Rousseau } \\
\text { Carol Couture } \\
\text { Manuel Vazquez }\end{array}$ \\
\hline $\begin{array}{l}\text { Resolução n } \\
24 / 2006\end{array}$ & Art. $\left.1^{\circ}, \mathrm{a}\right)$ & Teodore Schellenberg \\
\hline $\begin{array}{l}\text { Resolução n } \\
25 / 2007\end{array}$ & Art. $1^{\circ}, \S 1^{\circ}$ & $\begin{array}{l}\text { Teodore Schellenberg } \\
\text { Jean-Yves Rousseau } \\
\text { Carol Couture }\end{array}$ \\
\hline
\end{tabular}

\section{REFERENCIAS BIBLIOGRÁFICAS}

ARQUIVO NACIONAL. Dicionário brasileiro de terminologia arquivística. Rio de Janeiro: Arquivo Nacional, 2005. Disponible en Web: www.arquivonacional .gov.br. [Consulta em: 05/06/2014]

Diário Oficial da União. Lei no 8.159/1991, de 8 de janeiro de 1991.

Diário Oficial da União. Decreto no 4.073/2002, de 3 de janeiro de 2002.

Diário Oficial da União. Resolução no 5/ 1996, de 30 de setembro de 1996.

Diário Oficial da União. Resolução n ${ }^{\circ}$ 7/1997, de 20 de maio de 1997.

Diário Oficial da União. Resolução no 14/2002, de 24 de outubro de 2001.

Diário Oficial da União. Resolução no 20/2004, de 16 de julho de 2004.

Diário Oficial da União. Resolução n ${ }^{\circ}$ 24, de 3 de agosto de 2006.

Diário Oficial da União. Resolução no 25, de 27 de abril de 2007.

BROOKS, Philip Coolidge (1940). "The Selection of Records for Preservation", en The American Archivist,vol. 3, n.4, p. 221-234.

COLOMBO, F (1991). Os arquivos imperfeitos, memória social e cultura eletrônica. São Paulo: Editora Perspectiva. 
CONSELHO NACIONAL DE ARQUIVOS (Brasil) (2011). Câmara Técnica de Documentos Eletrônicos. E-ARQ Brasil: Modelo de Requisitos para Sistema Informatizados de Gestão Arquivística de Documentos. Rio de Janeiro: Arquivo Nacional.

COOK, Terry (1996). "Interacción entre la teoría y la práctica archivísticas desde la publicación del manual holandés en 1898." En: Actas del Congreso Internacional del Archivos, Pequim: ICA.

COOK, Terry (1998). "Arquivos pessoais e arquivos institucionais: para um entendimento arquivístico comum da formação da memória em um mundo pósmoderno", em Revista Estudos Históricos, vol.11, n. 21.

COOK, Terry (1999). Archival Appraisal and Colection: Issues, Challenges, New Approaches, Special Lecture Series, n. 21-22. Disponible en: www.mybestdocs.com/cpplt-nara-990421-2.html [Consulta: 06 de fevereiro de 2014].

COOK, Terry (2006). "Macroappraisal in Theory and Practice: Origen, Characteristics, and Implementation in Canada, 1950 - 2000.", en Archival Science, Springer.

COOK, Terry (2012). "Entrevista com Terry Cook para Revista InCID", em InCID, vol. 3, n. 2, pp. 142-156.

COUTURE, C. (2003). "La función valoración en la archivística contemporánea: una sinergia entre varias consideraciones complementarias", en Tabula, n. 6, pp. 23-49.

COX, R. J. "La valoración como un acto de memoria."Tabula, 2003, n. 6.

DELMAS, B.(2010). Arquivos para quê?Textos escolhidos. Ardaillon, Danielle (trad.) São Paulo: Instituto Fernando Henrique Cardoso.

DUCHEIN, M. (1993). "Prologo", en LLANSÓ I SANJUAN, J. Gestão de documentos: definición y análisis de modelos. Bergara: Departamento de Cultura del Gobierno Vasco, pp. 9-14.

DURANTI, L. (1994). "The Concept of Appraisal and Archival Theory", en American Archivist., vol. 57, Spring.

EASTWOOD, Terry (2003). "La valoración archivística en las sociedades democráticas", en Tabula: Revista de Archivos de Castilla y León, n.6, pp.75-85

FENOGLIO, Norma C (2013). Teoría de la macro evaluación de los documentos de archivo. Proyecto Evaluación de Documentos en Iberoamérica, Córdoba: Encuentro Grupo Editor.

FUGUERAS, R. A. (2003). Los archivos, entre la memoria histórica y la sociedad del conocimiento. Barcelona: Editorial UOC.

INTERNATIONAL COUNCIL ON ARCHIVES (1997). Committee on Eletrônic Records. Guide for managing electronic records from an archival perspective. Paris: ICA. Disponible en: www.ica.org [Consulta: 28 de janeiro de 2014]

INTERNATIONAL COUNCIL ON ARCHIVES. Dictionary. Disponible en: www.interpares.org. [Consulta: 27 de abril de 2014] 
INTERNATIONAL COUNCIL ON ARCHIVES. Appraisal Task Force Report Disponible en: www.interpares.org. [Consulta: 27 de abril de 2014]

INTERNATIONAL COUNCIL ON ARCHIVES. Multilingual Archival Terminology. Disponible en: www.ica.org/14282/multilingual-archival-terminology/multilingualarchival-terminology.html. [Consulta: 31 de janeiro de 2014]

JARDIM, J. M. (1995). “A Invenção da Memória nos Arquivos Públicos”, em Ciência da Informação. Brasília, vol. 25, n. 2.

JENKINSON, H. A. (1992). Manual of Archieve Administration. London: Percy Lund.

LAMB, W.K. (1962). El refinado arte de la destrucuión. Disponible en: www.mundoarchivistico.com/?menu=artículo\&accion=ver\&id=186 [Consulta: 04 de julho de 2014]

OLIVEIRA, D. A. (2013). "La evaluación funcional aplicada en los municipios brasileños", en Evaluación de Documentos en Iberoamérica. Córdoba: Encuentro Grupo Editor.

OLIVEIRA, E. B.; RODRIGUES, G. M. (2007). "O(s) conceito(s) de memória na bibliografia arquivística e nas práticas profissionais institucionalizadas no Brasil", en: Actas del Congresso de Archivologia do Mercosul, (Viña del Mar, 2007), Asociación de Archiveros de Chile.

POLLAK, M. (1989). "Memória, Esquecimento, Silêncio", en Estudos Histórico, vol. 2, n. 3 .

POLLAK, M.(1992). "Memória e identidade social", en Estudos Históricos, vol. 5, n. 10.

RIBEIRO, F.; SILVA, A. M. (2000). "A avaliação em arquivística: reformulação teórico-prática de uma operação metodológica", em Páginas $A \& B$., n. 5.

RIBEIRO, F.; SILVA, A. M. (2004). "A avaliação de informação: uma operação metodológica", en Páginas $A \& B$., n. 14.

RIBEIRO, F.; SILVA, A. M. (2009). "Perspectivar a avaliação como operação metodológica no âmbito da ciência da informação", en: Actas del Congreso Isko-España, n. ${ }^{\circ}$, , (Valência, 2009),ISKO.

ROUSSEAU, J. Y.; COUTURE, C. (1998). Os Fundamentos da Disciplina Arquivistica. Lisboa: Publicações Dom Quixote.

SCHWARTZ, J. M.; COOK, T. (2004). "Arquivos. Documentos e poder: a construção da memória moderna", em Revista do Arquivo Público Municipal de Indaiatuba, vol. 3, n. 3.

SILVA, A. M; RIBEIRO, F; RAMOS, J; REAL, M. L. (1999). Arquivística: teoria e prática de uma Ciência da Informação. Porto: Edições Afrontamento.

SCHELLENBERG, T.R. (2002). Arquivos Modernos:princípios e técnicas. Rio de Janeiro: FGV.

VÁZQUEZ MURILlO, M. (2006). Cómo seleccionar Documentos de Archivo. Buenos Aires: Alfagrama Ediciones. 
ANEXO 1 - Formulário de levantamento de dados

Universidade de Brasília - Brasil

Faculdade de Ciência da Informação

Programa de Pós-Graduação em Ciência da Informação

Aluna: Maria Ivonete Gomes do Nascimento

Este questionário objetiva obter informações a respeito da aplicação da legislação arquivística que trata da avaliação na Administração Pública Federal, assim como dos conceitos e das práticas adotadas na execução dos procedimentos para essa avaliação, nos ministérios do Poder Executivo Federal.

Nome do órgão:

Nome do/da responsável pelo arquivo:

Formação Profissional:

Nome do/da presidente ou representante da Comissão Permanente de Avaliação de Documentos no órgão:

Formação Profissional:

Nome do respondente:

Formação Profissional:

Data:

1 - Foi constituída, formalmente, a Comissão Permanente de Avaliação de Documentos no órgão?
( ) $\operatorname{sim}$
( ) não
( ) não sabe

2- Se sim, informe o instrumento legal:

3 - Se sim, informe a composição da Comissão Permanente de Avaliação de Documentos e a formação profissional de cada membro:

4 - Existe regimento interno da Comissão Permanente de Avaliação Documental?

( ) $\operatorname{sim}$ ( ) não ( ) não sabe

5 - A Comissão Permanente de Avaliação Documental se reúne com que frequência? 
6 - É realizado o trabalho de avaliação arquivística no órgão?

( ) $\operatorname{sim}$ ( ) não não sabe

7 - Se sim, existem normas que definam os procedimentos para o trabalho de avaliação arquivística?
( ) $\operatorname{sim}$
( ) não
( ) não sabe

8 - Para trabalho de avaliação, é feito o estudo da estrutura administrativa, das competências, das funções e das atividades das unidades?
( ) sim
( ) não
( ) não sabe

9 - Para o trabalho de avaliação, é feito o levantamento da produção documental?
( ) $\operatorname{sim}$
( ) não
( ) não sabe

10 - Se sim, relate como é desenvolvido esse trabalho:

11 - Para o trabalho de avaliação, é feita a análise do fluxo documental, tais como a produção e a tramitação dos documentos?
( ) $\operatorname{sim}$
( ) não
( ) não sabe

12-Para o trabalho de avaliação, são identificados os valores dos documentos, com base nos valores:
( ) administrativo
( ) legal
( ) fiscal
( ) técnico
( ) histórico
( ) outro
( ) não sabe
13 - Se outro, diga qual?

14 - Os documentos arquivísticos digitais recebem os mesmos procedimentos e critérios de avaliação que os documentos arquivísticos não digitais?
( ) sim
( ) não
( ) não sabe

15 - Se não, diga quais procedimentos e critérios adotam?

16 - Existe no órgão Sistema Informatizado de Gestão Arquivística de Documentos - SIGAD?
( ) sim
( ) não
( ) não sabe 
17- Se sim, em que momento são feitas a avaliação e seleção dos documentos?
( ) no armazenamento
( ) na captura
( ) na indexação
( ) na recuperação
( ) outro
( ) não sabe

18-Existe tabela de temporalidade e destinação relativa às atividades da área meio? Qual?

( ) do CONARQ

( ) não sabe

( ) outra, diga qual:

19 - Existe tabela de temporalidade e destinação relativa às atividades da área fim?

( ) $\operatorname{sim}$

( ) não

( ) não sabe

20- Se sim, essa tabela foi aprovada pelo Arquivo Nacional?
( ) $\operatorname{sim}$
( ) não
( ) não sabe

21 - Se sim, existe revisão periódica da tabela?
( ) $\operatorname{sim}$
( ) não
( ) não sabe

22- Existem documentos relativos às atividades-meio que não constem na tabela de temporalidade e destinação expedida pelo CONARQ?
( ) $\operatorname{sim}$
( ) não
( ) não sabe

23 - Se sim, essa tabela gerada foi aprovada pelo Arquivo Nacional?
( ) $\operatorname{sim}$
( ) não
( ) não sabe

24 - Houve eliminação de documentos no órgão?
( ) sim, acidental
( ) sim, planejada
( ) não( ) não sabe

25- É feito o registro dos documentos a serem eliminados em Listagem de Eliminação de Documentos?
( ) $\operatorname{sim}$
( ) não
( ) não sabe

26 - Se sim, a listagem de eliminação documental foi enviada ao Arquivo Nacional para aprovação?
( ) $\operatorname{sim}$
( ) não
( ) não sabe

27 - Se sim, quanto tempo decorreu do envio até a aprovação pelo Arquivo Nacional da listagem de eliminação de documentos? 
28 - Para a eliminação documental, é elaborado o Termo de Eliminação de Documentos?
( ) $\operatorname{sim}$
( ) não
( ) não sabe

29 - É feito Edital de Ciência de Eliminação de Documentos decorrente da aplicação da tabela de temporalidade e destinação?

( ) $\operatorname{sim}$

( ) não

( ) não sabe 